\title{
Algunas características anatómicas y acústicas de tres especies de angiospermas de Huayacocotla, Ver.
}

\author{
Alejandra Quintanar Isaías ${ }^{1}$ \\ Miguel de Icaza Herrera² \\ Laura Rivera Nava ${ }^{1}$ \\ Carmen de la Paz Pérez Olvera ${ }^{1}$
}

\begin{abstract}
RESUMEN
Se estudiaron algunas características anatómicas como el diámetro y longitud de los vasos, las dimensiones de las fibras y de los rayos. Asimismo, se obtuvieron las velocidades de sonido y los Módulos de Young $(Y)$ axial, radial y tangencial de tres especies de angiospermas colectadas en Huayacocotla, Veracruz. Los resultados indican que el número de poros/ $\mathrm{mm}^{2}$ y los grosores de la pared celular de las fibras influyen en la velocidad del sonido axialmente. Los rayos son estructuras que tienden a propiciar valores altos de velocidad de sonido radial y valores bajos en velocidad de sonido tangencial. Se recomienda a Prunus brachybotrya y Cleyera sp para sustituir especies de Acer, la que es empleada en la construcción de cajas de violines.
\end{abstract}

PALABRAS CLAVE:

Anatomía de la madera, ultrasonido, angiospermas, violines, Huayacocotla, México

\begin{abstract}
Some anatomical features of wood, like vessels, fibres and ray dimensions, were studied. Also axial and tangential sound velocity and Young's moduli, axial, radial and tangential were obtanied of three hardwood species from Huayacocotla, Ver. The results show that pore number, fibre cell wall thickness influence directly the axial sound velocity. Rays are structures which are partly responsible for high values of radial velocity and low values of tangential velocity. It is recommended that Prunus brachybotrya and Cleyera sp are used to substitute Acer species employed in box violin construction.
\end{abstract}

KEY WORDS:

Wood anatomy, ultrasound, hardwoods, violins, Huayacocotla, Mexico.

1 Departamento de Biología. Area de Botánica. Universidad Autónoma Metropolitana. México, D.F. C.P. 09340 México..

2 Instituto de Física, Universidad Nacional Autónoma de México. Circuito Exterior. Ciudad Universitaria. México, D.F. C.P. 04510 México.

Manuscrito recibido para su publicación el 11 de Diciembre de 1977 


\section{INTRODUCCION}

La madera es un tejido cuya composición celular le proporciona propiedades físicas y mecánicas especiales. Por su origen biológico, la constituyen células que presentan diversas formas, dimensiones y arreglos diferentes que van a caracterizar la estructura macroscópica y microscópica de las especies maderables. Asimismo, la abundancia de los diversos tipos celulares y sus contenidos ergásticos como cristales, gomas o taninos están relacionados con la respuesta física de este material al ser sometido a diferentes pruebas tecnológicas. La información derivada de los estudios anatómicos y físicos facilita la caracterización de cada especie y es de gran utilidad para sugerir criterios más sólidos que permitan establecer proyectos de silvicultura y mejoramiento genético de especies maderables.

En México existen especies forestales de gran potencial tecnológico (De la Paz y Quintanar, 1994; Quintanar y De la Paz, 1995) de las cuales pocas han sido estudiadas con métodos modernos. Uno de estos métodos es el acústico, para el cuál se emplea equipo que analiza el espectro natural de vibración (Orduña y Quintanar, 1992) o equipo que produce ondas mecánicas para medir sus características de propagación (Bucur, 1983).

La medición de propiedades acústicas permite la determinación de propiedades mecánicas en probetas de pequeñas dimensiones. Las constantes elásticas pueden utilizarse para formar clasificaciones normalizadas, comerciales, para su uso como material de construcción que requiera de buenos niveles de confianza, con lo que podrían ajustarse a estándares para garantizar productos de buena calidad.
La laudería, una disciplina artística, ha utilizado las ventajas de estos métodos para realizar pruebas con instrumentos antiguos, con lo que se ha abordado el problema de su calidad acústica (Hutchins, 1962; Haines, 1979) y en los que el tipo de madera que utilizaron para construirlos parece importar mucho. Schelleng (1982) ha señalado claramente que el constructor de violines, además de estar interesado como artesano en la buena trabajabilidad del material y como artista, en su belleza, debe, como músico, analizar su comportamiento acústico que podría ser el resultado de fenómenos relacionados con la estructura anatómica, la densidad del material, su capacidad de vibrar y amortiguar, o su rigidez, entre otros factores.

Ultimamente, se han impulsado estudios sobre cuáles son los aspectos de la anatomía de la madera relacionados con las propiedades acústicas en general (Rocaboy y Bucur, 1990), en donde se analizan las propiedades anatómicas y físicas de la madera con el objeto de resaltar aquellas, de entre las primeras, que correspondan con los valores más altos de las segundas.

Bucur (1995) presenta el estudio de la madera de varias especies de angiospermas templadas y algunas tropicales, destacando aquellas que presentan porosidad difusa y poca proporción de madera tardía representadas en fibras radialmente comprimidas. Entre estas destacan: Acer campestre, A. Pseudoplatanus y Populus spp (Tabla 1). Por otro lado, para Caesalpinia brasiliensis, que tiene la densidad más alta, fibras de paredes gruesas y mayor cantidad de extractivos, registra valores de la velocidad axial mayores a los del maple ( $A$. pseudoplatanus) y menores a los del Quercus petraea, lo que hace reflexionar sobre el impacto de las dimensiones de las paredes celulares y la presencia de cantidades importantes de extractivos. 
Asimismo, señala la importancia de las dimensiones de los rayos; entre más anchos son, los valores de la velocidad del sonido en la dirección tangencial (V. tangencial) decrecen y entre más altos son, los valores de la velocidad en dirección radial ( $\mathrm{V}$. radial) se incrementan (Tabla 1).

Rocaboy y Bucur (1990) estudiaron el comportamiento de las tapas de resonancia de los violines de Carleen Hutchins fabricados con madera de Picea excelsa para lo cual midieron las frecuencias naturales de los especímenes en un rango de $350-750 \mathrm{~Hz}$, obteniendo valores de velocidades de sonido, en sentido axial, desde $5085 \mathrm{~m} / \mathrm{s}$ hasta 5852 $\mathrm{m} / \mathrm{s}$, y en sentido tangencial desde 916 hasta $1188 \mathrm{~m} / \mathrm{s}$ y un Módulo de Young en dirección longitudinal de $189 \mathrm{~N} / \mathrm{m}^{2}$.

Bucur et al. (1991) estudiaron el comportamiento de la madera de reacción de algunas especies de gimnospermas encontrando que la velocidad de sonido en la madera tardía es siempre más alta que en la madera temprana; así como valores de velocidad ligeramente más bajos en las tres direcciones para la madera de compresión que las que se obtienen para madera normal. Las traqueidas en la madera de compresión son más cortas y presentan más cantidad de lignina. En la madera de tensión las fibras son más largas que las normales y contienen más celulosa, hemicelulosas y sustancias pécticas, por lo que los valores de sus velocidades de sonido son mayores. En este mismo estudio indican que en la madera juvenil, que es la que se forma en los primeros nueve años de vida del árbol, las velocidades se van incrementado gradualmente desde el anillo 1 hasta el 9. La composición química de las paredes celulares de este tipo de madera es en su mayor porcentaje de celulosa; posteriormente se incrementa el porcentaje de lignina y con esto disminuye, paulatinamente, la variabilidad de las velocidades acústicas en las diferentes direcciones.

Schelleng (1982) investigó 19 especies que se emplean para violines y encontró una relación muy interesante entre la densidad y las velocidades de sonido, y en general señala que entre más densa es una madera presenta una velocidad menor. Bucur y Chivers (1991),

Tabla 1. Velocidades axiales, radiales y tangenciales, así como las densidades de algunas especies estudiadas por Bucur (1995)

\begin{tabular}{||l|c|c|c|c||}
\hline ESPECIE & $\begin{array}{c}\text { V. AXIAL } \\
(\mathrm{m} / \mathrm{s})\end{array}$ & $\begin{array}{c}\text { V. RADIAL } \\
(\mathrm{m} / \mathrm{s})\end{array}$ & $\begin{array}{c}\text { V.TANGENCIAL } \\
(\mathrm{m} / \mathrm{s})\end{array}$ & $\begin{array}{c}\text { DENSIDAD } \\
\mathrm{kg} / \mathrm{m}^{3}\end{array}$ \\
\hline Acer campestre & 4695 & 2148 & 1878 & 623 \\
Acer pseudoplatanus & 4350 & 2590 & 1914 & 700 \\
Aesculus hippocastanum & 4782 & 2311 & 1383 & 510 \\
Caesalpinia brasiliensis & 4935 & 3435 & 2034 & 932 \\
Fagus sylvatica & 5074 & 2200 & 1560 & 674 \\
Liriodendron tulipifera & 5625 & 2047 & 1511 & 574 \\
Quercus petraea & 5071 & 2148 & 1538 & 600 \\
Populus spp & 5074 & 2178 & 1646 & 326 \\
Platanus acerifolia & 5060 & 2190 & 1640 & 620 \\
\hline
\end{tabular}


investigaron el comportamiento de especies australianas y europeas y encontraron que, en general, presentan propiedades acústicas muy similares, calculadas a partir de las velocidades del sonido. En general, plantean que a pesar de las bajas densidades en las coníferas, respecto de las angiospermas, los valores altos de las velocidades de sonido en sentido axial parecen estar relacionados con las longitudes de las traqueidas que, en general, son más largas que las fibras en las angiospermas.

\section{OBJETIVO}

El objetivo de este trabajo fue estudiar las características anatómicas y acústicas de tres maderas mexicanas de clima templado: Prunus brachybotrya, Cleyera sp. y Clethra mexicana.

Para las especies de este trabajo, Hoadley (1989) incluye a las maderas de dos de estos géneros con pesos específicos de 0.45 para Clethra y 0.50 para Prunus, que de acuerdo con la Asociación Internacional de Anatomistas de la Madera (IAWA, 1989) son ligeras; para Cleyera, no existe información.

\section{METODOLOGIA}

El material estudiado proviene de tres árboles colectados en la región de Huayacocotla, Veracruz. Cada especie está representada por un árbol; los ejemplares de herbario se encuentran depositados en el Herbario Metropolitano de la Universidad Autónoma Metropolitana Iztapalapa. Estos árboles tenían fustes rectos, con diámetros a la altura del pecho de $25 \mathrm{~cm}$ y alturas de 8 a $10 \mathrm{~m}$. Cada árbol se seccionó en trozas de $1 \mathrm{~m}$ de largo y de la primera troza se obtuvieron rodajas de $3.5 \mathrm{~cm}$ de grueso. De estas rodajas se cortaron cubos de $2 \mathrm{~cm}$ de arista para las preparaciones de cortes típicos y material disociado con el objeto de medir los caracteres anatómicos cuantitativos (Johansen, 1940; Kukachka, 1977). De las mismas rodajas, se obtuvieron paralelepípedos rectangulares sobre los que se midieron las velocidades de propagación de las ondas longitudinales en las tres direcciones fundamentales, para con esto, calcular los valores de las propiedades dinámicas (Fig. 1A y 1B). Para obtener los valores anatómicos cuantitativos se describió y midió en las caras transversales la porosidad, el número y el diámetro tangencial de poros. En las caras tangenciales se midió la longitud de los vasos, el número, la altura y la anchura de los rayos. En el material disociado se midió la longitud, el diámetro y el grosor de la pared celular de las fibras y se verificó la longitud de los elementos de vaso. La denominación de los caracteres se hizo con base en las clasificaciones sugeridas por Chattaway (1932) y los Comités de la IAWA (1937, 1939 y 1989).

Para la determinación de la velocidad del sonido, se obtuvo, por un lado, la densidad de los paralelepípedos y por otro el tiempo de barrido. La densidad se calculó pesando y midiendo las dimensiones de los especímenes y el tiempo, con el auxilio de un osciloscopio conectado a un amplificador, a un generador y a un activador de pulsos. A este osciloscopio estaban conectados dos transductores idénticos que sirvieron de emisor y de receptor. El emisor hace el papel de una "bocina", mientras que el receptor, de un "micrófono". Las ondas mecánicas incidentes sobre el disco de acero del receptor son transmitidas al disco de cerámica, que compone la parte interna del transductor, deformándolo, lo que da lugar a un campo eléctrico, y por lo tanto a una diferencia de potencial entre sus electrodos, que se hace visible mediante el osciloscopio, lo cual permite observar los desplazamientos microscópicos del disco de acero del transductor. De esta manera, se puede medir el tiempo que se tarda la onda en 

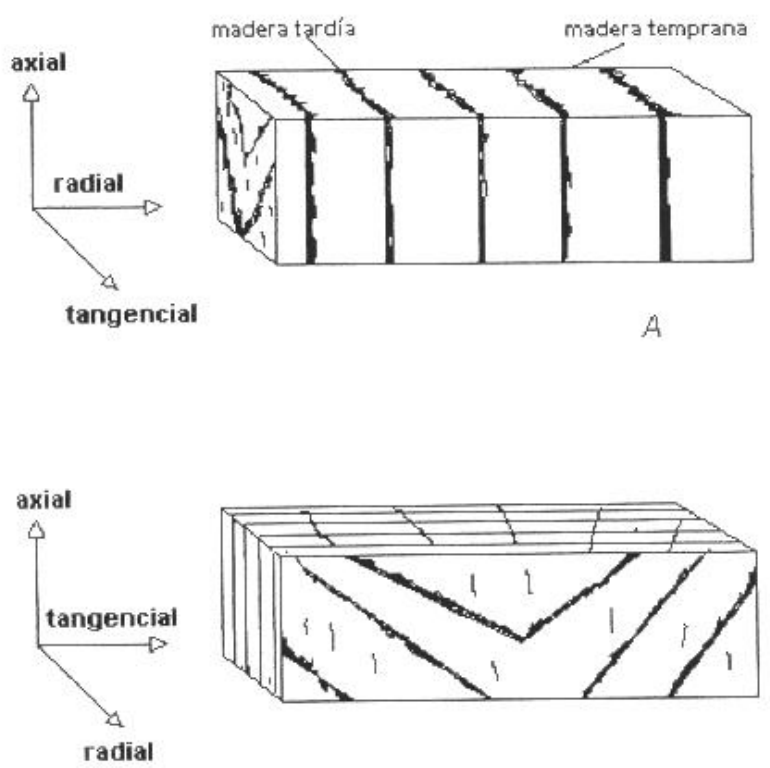

radial

B

Figura 1. Paralelepípedos de madera usados para medir velocidades de sonido en sentido axial, radial y tangencial. A. Probeta en sentido radial. B. Probeta en sentido tangencial

llegar, desde que es emitida por el emisor, hasta que es captada por el receptor. Con estos datos, se calcula la velocidad del sonido, mediante el cociente de la distancia recorrida entre el tiempo empleado.

La pantalla del osciloscopio es un sistema de coordenadas sobre el que se traza la diferencia de potencial (eje vertical) y el tiempo (eje horizontal). La calibración del osciloscopio involucra la amplificación, es decir el número de volts que representa una división vertical y la velocidad de barrido (tiempo que toma una división horizontal), de manera que el tiempo se calcula multiplicando el número de divisiones de la pantalla del osciloscopio por el tiempo de barrido y entonces la velocidad será la longitud de la probeta entre el tiempo calculado (fig. 2). Estas ondas longitudinales son producidas por un pulso que hace que el material piezo-eléctrico que forma el medio elástico del transductor (la cerámica), se mueva de manera paralela a la dirección de la onda, por lo que es una velocidad en sentido axial, producida por ondas longitudinales y como viaja en sentido axial, se ha abreviado VLA. Por otro lado, como la madera es un material anisotrópico, fue necesario medir este mismo tipo de velocidades en sentido tangencial y en sentido radial, siendo sus abreviaciones, respectivamente, VLT y VLR. Con las velocidades de sonido y las densidades se calculó el Módulo de Young ( $Y$ ) para cada pieza de madera, de acuerdo con la ecuación: 


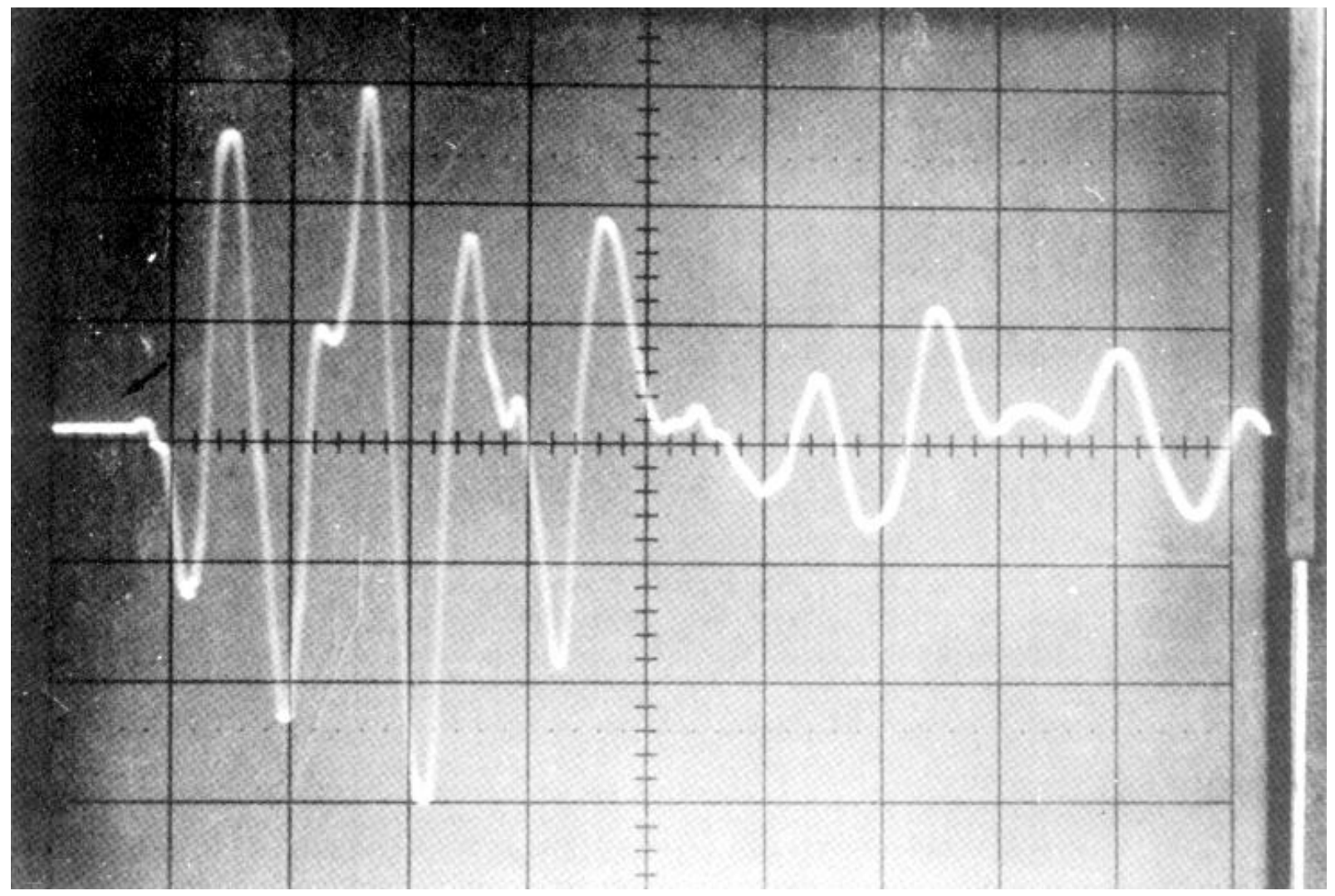

Figura 2. Señal del pulso en el osciloscopio. La distancia horizontal es el tiempo que tarda en recorrer el pulso dentro de la probeta, ya sea en sentido radial o tangencial.

$$
c_{l}=Y / \rho
$$

donde $\mathrm{c}_{\mathrm{l}}$ es la velocidad del sonido producida por ondas longitudinales, $Y$ es el Módulo de Young y $\rho$ es la densidad de la madera.

\section{RESULTADOS Y DISCUSION}

Las especies estudiadas presentan una estructura microscópica que puede explicar algunas de las respuestas acústicas que se han encontrado. Cuando se inducen ondas mecánicas en una muestra de madera (Bucur, 1995), orientada en la dirección axial, algunos componentes de las mismas viajan con menor o mayor dificultad. De esta manera, la madera se comporta como un filtro en el que las paredes celulares proveen un camino continuo para la onda, que probablemente choca con los extremos de las fibras o vasos. El avance de la onda puede ser dificultado o facilitado, dependiendo de la presencia o ausencia de extractivos, por el tamaño de lúmenes y por los espesores de la pared celular.

Tal vez si se pensara en una reconstrucción ultramicroscópica donde se observara cómo se rompe la onda (que en términos de energía se traduce en disipación) se podría establecer una relación donde con estructuras sencillas, 
esto es, con sólo dos tipos celulares se facilitaría el paso de la onda ; éste podría ser el caso de las coníferas; sin embargo, las especies de este estudio, debido a su diversidad celular tienen patrones anatómicos más complejos, esto es, elementos de vaso, fibras $\mathrm{y} / \mathrm{o}$ fibrotraqueidas, parénquima axial y rayos. Por esta situación, resulta interesante presentar de manera comparativa los valores anatómicos con los acústicos que se estudiaron (Tabla 2).
De la Tabla 2 se observa lo siguiente: las velocidades axiales son más grandes que las velocidades radiales y las tangenciales. De entre estas últimas dos, los valores en sentido radial son más altos con respecto a los tangenciales. En general, la obtención de valores más altos para las velocidades axiales se explica por el hecho de que el viaje de las ondas se realiza a través de los elementos verticales, el que sólo es interrumpido por las terminaciones de las fibras (o fibrotraquiedas) y de los vasos. De

TABLA 2. Valores anatómicos y acústicos de las especies estudiadas *Valores dados en Micras

\begin{tabular}{||l|c|c|c||}
\hline \multicolumn{1}{|c|}{ CARACTER / ESPECIE } & $\begin{array}{c}\text { Prunus } \\
\text { brachybotrya }\end{array}$ & Cleyera sp & $\begin{array}{c}\text { Clethra } \\
\text { mexicana }\end{array}$ \\
\hline Número de poros $/ \mathrm{mm}^{2}$ & 31 & 42 & 56 \\
\hline Diámetro de vasos & 53 & 56 & 51 \\
\hline Longitud de vasos & 377 & 956 & 622 \\
\hline Anchura poliseriados & & 19 & 32 \\
\hline Altura poliseriados & 82 & 1174 & 562 \\
\hline Número de rayos poliseriados $/ \mathrm{mm}$ & 2 & 12 & 8 \\
\hline Número de rayos uniseriados/mm & 8 & - & - \\
\hline Altura de rayos uniseriados* & 280 & - & - \\
\hline Anchura de rayos uniseriados* & 17 & - & - \\
\hline Longitud de fibras* & 410 & - & - \\
\hline Longitud de fibrotraqueidas & - & 1030 & 748 \\
\hline Grosor de pared celular* & 6.6 & 8 & 4.4 \\
\hline Diámetro de fibra o fibrotraqueida* & 20 & 14 & 28 \\
\hline Velocidad axial $(\mathrm{VLA}) \mathrm{m} / \mathrm{s}$ & 4790 & 4680 & 4384 \\
\hline Velocidad radial $(\mathrm{VLR}) \mathrm{m} / \mathrm{s}$ & 2167 & 2401 & 1443 \\
\hline Velocidad tangencial $(\mathrm{VLT}) \mathrm{m} / \mathrm{s}$ & 1293 & 1654 & 1069 \\
\hline$Y$ - axial $\left(\times 10^{8} \mathrm{~N} / \mathrm{m}^{2}\right)$ & 160 & 150 & 109 \\
\hline$Y$ - radial $\left(\times 10^{8} \mathrm{~N} / \mathrm{m}^{2}\right)$ & 32 & 40 & 13 \\
\hline$Y$ - tangencial $\left(\times 10^{8} \mathrm{~N} / \mathrm{m}^{2}\right)$ & 12 & 19 & 6 \\
\hline Densidad $\left(\mathrm{kg} / \mathrm{m}^{3}\right)$ & 692 & 683 & 567 \\
\hline VLA/VLR & 2.2 & 1.9 & 3 \\
\hline VLA/VLT & 3.7 & 2.8 & 4.1 \\
\hline \hline
\end{tabular}


acuerdo con la literatura (Bucur 1995, Schelleng 1982, Bucur y Chivers, 1991), los valores de las velocidades axiales siempre son más altos y llegan a ser aproximadamente del doble que los valores en las direcciones radiales y un poco más que en las tangenciales y sus variaciones dependerán de los métodos empleados para determinarlos, por ejemplo para Acer pseudoplatanus, Bucur (1995) registra una velocidad de $4350 \mathrm{~m} / \mathrm{s}$ y Schelleng de $3500 \mathrm{~m} / \mathrm{s}$.La madera de las especies aquí estudiadas presentó algunas características anatómicas que pueden estar influyendo tanto en la transmisión de la velocidad del sonido, así como en los valores de $Y$.

La transmisión de las ondas de sonido en sentido axial puede depender del número de poros por milímetro cuadrado. En estas especies se observa claramente que Clethra tiene el mayor número de poros, lo que la hace una especie con mayores espacios, esto es, más porosa. Le sigue Cleyera y en seguida, Prunus; consecuentemente sus velocidades son mayores para esta última y menores para la primera; asimismo, resalta que para las tres especies el diámetro de los vasos es muy similar.

Aparentemente la longitud de vasos y fibras no es una característica que esté proporcionalmente relacionada con la transmisión de la velocidad del sonido en sentido axial, lo que se observa al comparar los valores de las VLA de las tres especies, donde $C$. mexicana tiene las longitudes de vasos y fibras intermedias y los valores más bajos de VLA. El valor promedio de la velocidad radial (VLR) es mayor para Cleyera, la que presenta la longitud de vasos y fibras más largas, así como los rayos más altos. En este sentido, los rayos funcionan como tubos horizontales, que al ser más altos y más abundantes, permiten una mejor transmisión del sonido, lo que lógicamente está relacionado con lo propuesto en la Teoría del Continuum, donde la madera se representa como un sistema rectangular de tubos axialmente similares, embebidos en una matriz de lignina $y$ en el cual, la orientación longitudinal de las fibras puede ser parcialmente perturbada por tubos horizontales o rayos que, dependiendo de su anchura y altura influirán en el valor de sus velocidades. Asimismo, es posible que estructuras con porosidades difusas con porciones pequeñas de madera tardía en los anillos de crecimiento estén más cercanas a la estructura que propone esta teoría y estén más alejadas las que poseen porosidad circular, que es lo que ocurre con algunas angiospermas (Bucur,1987).

Los valores de las velocidades tangenciales (VLT) se espera que sean menores, debido al hecho que las ondas deben atravesar las paredes celulares, tanto de elementos horizontales, como verticales. Los valores menores de velocidad los presentó Clethra, en la que la gran cantidad de espacios influye en la pérdida de energía, esto es, presenta número de poros y diámetro de lúmen de las fibrotraqueidas mayores, así como paredes más delgadas. Por otro lado, Prunus brachybotria presenta una VLT intermedia, con la mayor anchura de rayos, diámetro y grosor de paredes de las fibras medianas y Cleyera presenta los diámetros de las fibrotraqueidas más pequeños y las paredes más gruesas. Esto puede explicar el valor menor de VLT en P. brachybotrya en comparación con el más alto de Cleyera (Tabla 2). Al comparar las velocidades axiales con las señaladas por Bucur (Tabla 1) se observa que tanto $P$. brachybotrya como Cleyera, tienen valores de VLA y VLR parecidos a los de Acer campestre y A. pseudoplatanus.

En la Tabla 2 se observan los cocientes de VLA/VLR y VLA/VLT. Estos cocientes reflejan las características anisotrópicas, de manera que el primer cociente tiende a generar valores menores que el segundo; esto está relacionado con la estructura transversal-radial (Fig 1A). E 
segundo cociente presenta valores mayores debido a la estructura transversal-tangencial (Fig. 1B) Esto se debe a las dimensiones de los rayos, a los grosores de las paredes celulares y a los espacios generados por la cantidad de poros y anchura de lúmenes de las fibras (Fig. 3a-i). Estos cocientes podrían ayudar a entender el criterio de porosidad, homogeneidad $y$ heterogeneidad no anatómica a la que se refiere la Teoría del Continuum (Bucur, 1987). Un valor cercano a uno, de cualquier cociente, significaría que el material es más homogéneo, por lo que, entre más grande es su valor, entonces el material es por supuesto más anisotrópico, esto es, más heterogéneo. Tanto Cleyera como $P$. brachybotrya son especies menos heterogéneas, aunque la última presenta valores de $Y$ mayores a los de Clethra.

Los cocientes de las dos primeras especies son muy parecidos a los citados para las especies de Acer, que han sido muy empleadas en la construcción del cuerpo de los violines.

\section{CONCLUSIONES}

Existe un conjunto de patrones y características celulares que son más eficientes para transmitir el sonido y otras que pueden amortiguarlo. En el primer aspecto, esto es, de la eficiencia, se puede resumir que en términos comparativos para estas tres especies, las que más se parecen a aquellas empleadas para las cajas de resonancia son Cleyera sp. y $P$. brachybotria, lo que se refleja en buena medida en el grosor de las paredes de las fibrotraqueidas 0 fibras $y$ en el menor número de poros, lo que a su vez está relacionado directamente con la densidad. En términos de una estructura amortiguadora, esto es, en la que el número de poros por $\mathrm{mm}^{2}$ es mayor, con paredes delgadas y diámetro de lúmenes de fibras grande, como es el caso de Clethra, los valores de velocidad de sonido, en los tres sentidos son menores, lo que hace que ésta sea una especie no recomendable para esta parte del violín.

Por otro lado, en cuanto a los valores de los módulos de Young ( $Y s$ ), Prunus y Cleyera los tienen más altos, por lo que se podrían ubicar en el mismo grupo de Acer. Bucur (1995) presenta para especies de Acer, valores de $Y$ en sentido axial de 150 y $160 \times 10^{8} \mathrm{~N} / \mathrm{m}^{2}$, lo que sugiere un comportamiento muy similar de $P$. brachybotrya y Cleyera con este género. Clethra tiene los valores menores por lo que se podría recomendar para usos más relacionados con el tallado y modelaje.

De los resultados, obtenidos se puede resumir, que existen en México especies con características anatómicas y acústicas que pueden sustituir aquellas de importación y que a través de proyectos silviculturales serios es posible promover especies que tienen tamaños y dimensiones celulares óptimos, con las cuales proveer al mercado nacional de maderas de alta calidad para usos tan específicos como la elaboración de instrumentos musicales $u$ otros que requieren de otras especificaciones.

\section{RECONOCIMIENTOS}

Este trabajo se realizó gracias al apoyo de varias personas e instituciones. Al Ing. Raul de la Cadena por su apoyo en la recolección; al M. en I. Víctor Ordóñez y al Dr. Raymundo Dávalos, del Instituto de Ecología, A. C. por el apoyo en la elaboración de las muestras y la revisión de algunos conceptos teóricos previos. Al Dr. Victor Castaño por el apoyo brindado con el equipo del Laboratorio de Ultrasónica del Instituto de Física, UNAM. Por la revisión del manuscrito al Biól. Gerardo López y al Dr. Alfonso Torre-Blanco. Por el apoyo económico a la División de Ciencias Biológicas y de la Salud de la Universidad Autónoma Metropolitana-Iztapalapa 

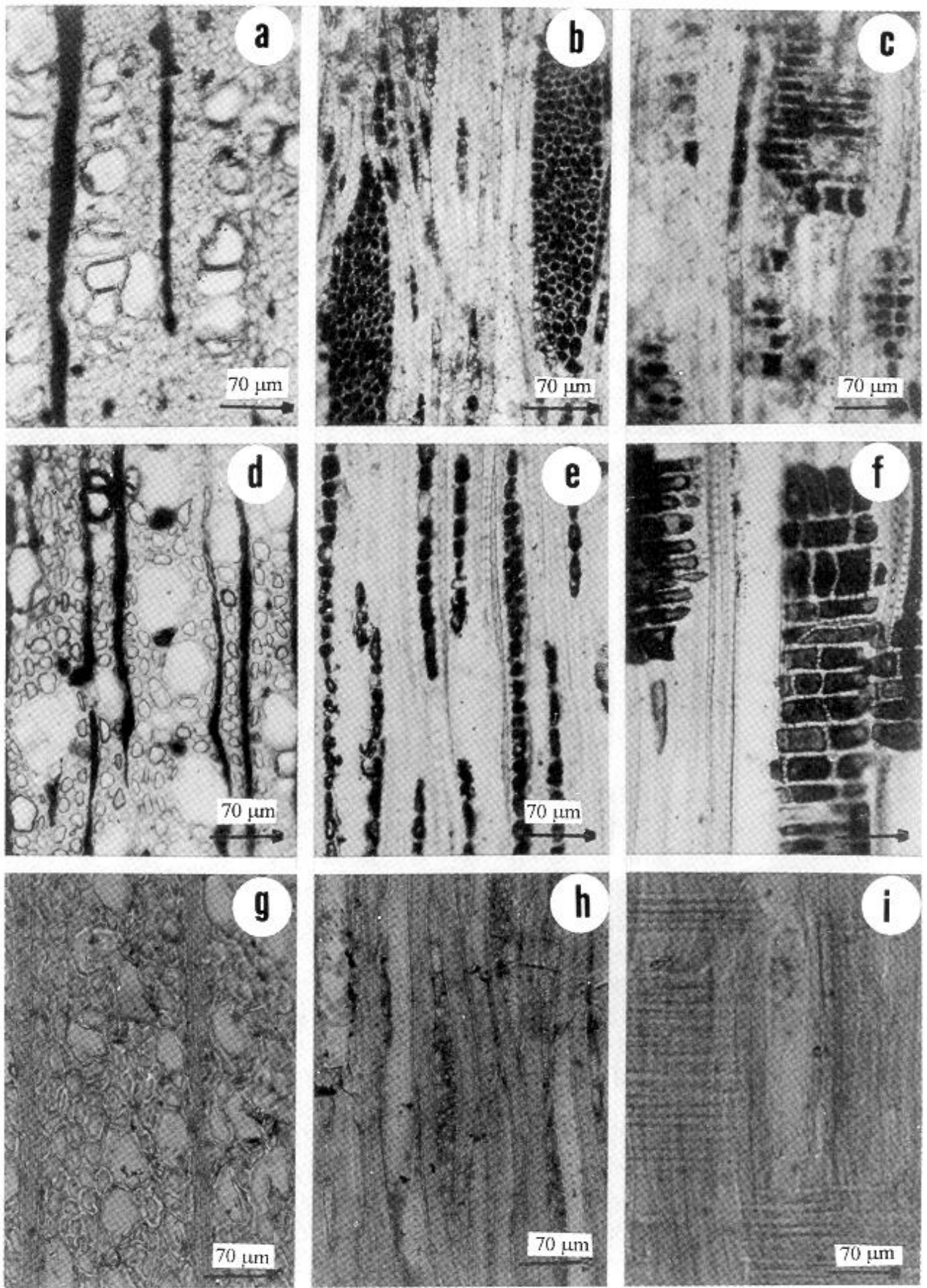

Figura 3. Cortes transversales (izquierda), tangenciales (centro) y radiales (derecha). a-c: Prunus brachybotrya; d-e: Cleyera sp; f-h: Clethra mexicana 


\section{REFERENCIAS}

Bucur, V. 1983. An ultrasonic method for measuring the elastic constants of wood increment cores bored from living trees. Ultrasonics may. 116126.

Bucur, V. 1987 Wood structural anisotropy estimated by acoustics invariants. IAWA Bulletin n.s. 9(1):67-79.

Bucur, V. y Chivers, R. C. 1991. Acoustic properties and anisotropy of some australian wood species. Acustica 75:69-74.

Bucur, V., G. Janin, C. Herbe y J. M. Ory 1991. Ultrasonic detection of reaction wood in european species. 10 e Congres Forestier Mondial 1726 septembre. Paris, France.

Bucur, V. 1995. Acoustics of Wood. CRC PRESS, New York.

Chattaway, M. 1932. Proposed standards for numerical values used in describing woods. Trop. Woods, 29:20-28.

De la Paz P. O., C. y A. Quintanar I. 1994. Características anatómicas de la madera de cinco especies del Estado de Jalisco, México. Acta Botánica Mexicana 27:75-87.

Haines, D. 1979. On musical intrument wood. I. Catgut Acoust. Soc. Newslett. 31:23-32.

Hoadley, R. 1989. Understanding wood. Tauton Press. USA. 256 pp.

Hutchins, C. M. 1962. The physics of violins. Scientific American. november, 79-93.
IAWA Committee. 1937. Committee on the standarization of terms of cell size. Standard terms of length vessel members and wood fibers. Trop. Woods 51:21.

IAWA Committee. 1939. Committee on the Nomenclature. Standard terms of size for vessel diameter and ray width. Trop. Woods 59:51-52.

IAWA Committee. 1989. List of microscopic features for hardwood identification. IAWA Bull. n.s. 10:219-232.

Johansen, D. A. 1940. Plant Microtechnique. McGraw Hill, New York. $253 \mathrm{p}$.

Kukachka, B. F.1977. Sectioning refractory woods for anatomical studies. USDA. Forest Service Research Note FLP-0236. Madison, Wisconsin. $9 \mathrm{p}$.

Orduña, F. y A. Quintanar. 1992. A preliminary determination of the mechanical properties of four species of tropical wood from Mexico. Journal of Sound and Vibration 154(2):365-368.

Quintanar I., A. y C de la Paz P.O. 1995. Anatomía de la madera de ocho especies de angiospermas. Boletín de la Sociedad Botánica de México 58:5-14.

Rocaboy, F. y V. Bucur. 1990. About the physical properties of wood of twentieth century violins. Catgut Acoust. Soc. J. (Series II) 6:21-28.

Schelleng, J. C. 1982. Wood for violins. Catgut Acoust. Soc. Newslett. 37:819. 